\title{
A Review of a Diagnostic Tool: Galactomannan
}

\section{Gulhadiye Avcu', Deniz Yilmaz Karapinar ${ }^{2 *}$}

'Ege University Faculty of Medicine, Children's Hospital, Department of Pediatric Infectious Disease, 35040 Bornova Izmir, Turkey

${ }^{2}$ Ege University Faculty of Medicine, Children's Hospital, Pediatric Hematology, 35040 Bornova Izmir, Turkey

\section{Article Info}

\section{Article Notes}

Received: August 23, 2018

Accepted: October 23, 2018

\section{${ }^{*}$ Correspondence:}

Dr. Deniz Yilmaz Karapinar, Ege University Faculty of

Medicine, Children's Hospital, Pediatric Hematology, 35040

Bornova Izmir, Turkey; Email: dyilmazk@yahoo.com.

C 2018 Karapinar DY. This article is distributed under the terms of the Creative Commons Attribution 4.0 International License.

\section{Keywords:}

Invasive aspergillosis

Galactomannan antigen

Serum

Bronchoalveolar lavage fluid

\section{ABSTRACT}

Invasive fungal infections, including invasive aspergillosis are associated with a high morbidity and mortality especially in immunocompromised patients. Diagnosis is often difficult due to several factors such as delay in clinical suspicion and the lack of spesific clinical findings. Galactomannan is a polysaccharide cell wall component of Aspergillus and galactomannan antigen detection has become widely used for diagnosis of invasive aspergillosis. Here, we tried to discuss the diagnostic value of the galactomannan test in the context of literature review.

\section{Introduction}

Invasive fungal infections (IFI) have been increasing in neutropenic adult and pediatric patients. Invasive Aspergillosis (IA) became the most common form of IFI after the introduction of fluconazole prophylaxis ${ }^{1,2}$. It is also the most significant opportunistic fungal infection following hematopoietic stem cell transplantation (HSCT) which causes a 25-35\% mortality despite the new antifungal agents such as voriconazole and echinocandins $s^{3,4,5}$. Early diagnosis of IA play an important role in the patients' survival outcome. The diagnosis of IA is often difficult because both the clinical and radiological findings are often non-specific. A high index of suspicion is required to successfully achieve a positive diagnosis. İnvasive procedures such as tissue biopsy and bronchoalveolar lavage (BAL) can not always be done due to the clinical condition of the patients ${ }^{6}$. The Platelia (Sanofi Diagnostic Pasterur, Marnes la Coquette, France) enzyme immunoassay (EIA) for the detection of galactomannan (GM) is currently one of the more used methods. The GM assay was approved by the Center for Devices and Radiological Health, Food and Drug Administration (FDA), in 2003 for the testing of serum samples as an aid to the diagnosis of IA.

Galactomannan is a polysaccharide that is a major constituent of Aspergillus cell walls. It is a soluble, heat stable antigen released by especially Aspergillus species during active cell replication (during hyphal growth). Circulating GM can be detected in serum or plasma and may be present in BAL- fluid, cerebrospinal fluid (CSF) or other body fluids ${ }^{11}$. The GM EIA is performed with an optical read-out that is interpreted as a ratio relative to the optical density (OD) of a threshold control provided by the manufacturer; this ratio is called the OD index. The FDA has a suggested threshold OD index of 0.5 and an OD index $\geq 0.5$ is generally considered to be a positive result.

The European Organization for Research and Treatment of Cancer/Mycosis Study Group (EORTC/MSG) guidelines recommend 
detection of GM as a standalone microbiological criterion for diagnosis of probable patients in association with clinical and host factors. GM testing allows the early diagnosis of aspergillosis and the prompt initiation of antifungal therapy ${ }^{7,8}$. The new guidelines of the International Pediatric Fever and Neutropenia Guideline Panel also recommend considering prospective monitoring of serum GM twice weekly in high-risk hospitalized children, for early diagnosis of IA [recommendation strength is moderate (2B) ] ${ }^{9}$. The published guidelines of the Fourth European Conference on Infections in Leukemia (ECIL4) recommend prospective monitoring, twice weekly, for early diagnosis of invasive aspergillosis in children at highrisk (strength of $\mathrm{A}-\mathrm{II})^{10}$.

\section{GM Testing in Serum}

GM antigen has become an important diagnostic tool in the management of patients at risk for IA. GM can be detected in the serum in some patients before the presence of clinical signs or symptoms of IA. Monitoring for GM can be used in neutropenic patients who have a relatively high probability ( $>5-10 \%$ ) of developing IA such as; Acute myeloid leukemia (AML) and myelodysplastic syndrome during intensive chemotherapy and patients at the early engraftment phase of the allogeneic HSCT. GM screening every 3-4 days in these groups can increase the detection rate of IFI and decrease the costs of antifungal drugs ${ }^{12,13}$. A single positive GM index of $\geq 0.7$ or two consecutive samples of $\geq 0.5$ should prompt a diagnostic work-up ${ }^{10}$. GM monitoring can be continued after antifungal therapy has been initiated. Several studies have shown that the course of the GM index during antifungal therapy is predictive of the outcome ${ }^{14}$. Persistent GM antigenemia during therapy is a poor prognostic sign and should prompt clinical reassessment. Mortality in IA patients with persistently elevated serum GM was reported to be higher than in those with negatively converted serum GM during antifungal therapy ${ }^{15,16}$.

The specificity and sensitivity of the assay in serum samples varies across the different clinical studies at different cut-off points of the galactomannan index. The sensitivity of GM testing varies in the range of $30-100 \%$, while specificity has been reported to be $>75 \%{ }^{17}$. A meta-analysis showed the efficacy of the GM test in case of proven IA had an overall sensitivity and specifity of $71 \%$ and $89 \%$ respectively ${ }^{17}$. The combined sensitivity and specificity of the five pediatric studies that had been considered adequate by the International Pediatric Fever and Neutropenia Guideline Panel, 0.76 (95\% CI, 0.62 to 0.87 ) and 0.86 (95\% CI, 0.68 to 0.95 ), respectively ${ }^{9}$. A retrospective pediatric study reported 0.91 sensitivity and 0.81 specificity; while the false positive rate was $18.3 \%^{18}$.

A recent meta-analysis reported by Lehrnbecher et al. ${ }^{9}$ showed that GM negative predictive values were high, ranging from $85 \%$ to $100 \%$ for screening and $70 \%$ to $100 \%$ in the diagnostic setting, but failure to identify nonAspergillus molds limits its usefulness. Among 10 studies including 100 patients in which serum GM was used as a screening test for IFI during neutropenia or post-HSCT, specificity ranged $50 \%-100 \%$, sensitivity $0 \%-100 \%$, positive predictive value $0 \%-100 \%$, and negative predictive value $85 \%-100 \%$. Among 84 patients (in children with symptoms potentially suggestive of IFI) from 8 studies in which GM testing performed as a diagnostic test; specificity ranged $35 \%-100 \%$, sensitivity $14 \%-100 \%$, positive predictive value $0 \%-100 \%$, and negative predictive value 70\%-100\%. Different age and characteristics of patient populations of these studies results may contribute wide range. Additionally, most of the studies eating habbits of the patients, and antibiotic usage or prophylaxis against fungal infetions were not discussed. They may have also effect on the results. There is a great necessity for a prospective study including children age who are on standardized antibiotic and antifungal therapy.

Serial GM testing is recommended both for early diagnosis and the follow-up of patients with IA as a prognostic marker to evaluate the course of the disease and the response to antifungal treatment ${ }^{19}$. Han et al. ${ }^{20}$ investigated serum GM index for early prediction of mortality in immunocomromised children with invasive pulmonary aspergillosis. They found out that, serum GM levels were higher in the fatality group than in the survival group during the entire period of antifungal therapy, and serum GM at 1 week after antifungal therapy was most significantly associated with mortality. A serum GM $>1.50$ at 1 week after antifungal therapy indicated a sensitivity and specificity of $61.5 \%$ and $89.3 \%$, respectively, in predicting mortality within 12 weeks after antifungal therapy. They suggested more intensive antifungal therapy in patients at high risk for mortality, who show a persistently high level of serum GM within 1 week of antifungal therapy. Most of the studies recommended once to twice weekly sampling as in the 27 studies included in the meta-analysis by Pfeiffer et al. ${ }^{1721-23}$. In Pfeiffer's ${ }^{17}$ meta-analysis (children and adults), the range of sensitivity and specificity varied between $38 \%$ and $100 \%$, and between $17 \%$ and $100 \%$, respectively.

Gefen et al. ${ }^{24}$ researched the serial serum GM screening for IA diagnosis in children after stem cell transplantation and with high risk leukemia prospectively. GM sensitivity and specificity were 0.8 and 0.66 , respectively; positive- and negative-predictive values were 0.22 and 0.96 , respectively. The rate of false positive cases was high (\%78).

GM detection in the serum of non-neutropenic patients has limited importance because of its limited sensitivity ${ }^{25}$. A meta-analysis including 27 studies reported an overall sensitivity of serum galactomannan assay of $71 \%$ and specificity of $89 \%$. However, when oncohematological 
Table 1. The comparison of the results of studies on galactomannan.

\begin{tabular}{|l|l|c|c|c|}
\hline \multicolumn{1}{|c|}{ study } & \multicolumn{1}{c|}{ patient group } & sample type & sensitivity & specifity \\
\hline Pfeiffer CD, et al. (2006) & Adult, neutropenic & serum & $30-100 \%$ & $>75 \%$ \\
\hline Lehrnbecher T, et al. (2012) & Pediatric, neutropenic & serum & $0 \%-100 \%$ & $50 \%-100 \%$ \\
\hline Gefen A, et al. (2015) & Pediatric, neutropenic & serum & $80 \%$ & $66 \%$ \\
\hline Zhou W, et al. (2017) & Adult, Non-neutropenic & serum & $37.8 \%$ & $87.1 \%$ \\
\hline Zhou W, et al. (2017) & Adult, Non-neutropenic & BAL & $75.6 \%$ & $80.7 \%$ \\
\hline Bergeron A, et al. (2010) & Adult, neutropenic & BAL & $57.6 \%$ & $95.6 \%$ \\
\hline Desai R, et al. (2009) & Pediatric Neutropenic+non-neutropenic & BAL & $78 \%$ & $92 \%$ \\
\hline
\end{tabular}

patients were excluded from the analysis, the sensitivity and specificity of the test dropped to $22 \%$ and $84 \%$, respectively ${ }^{17}$. In a recent prospective study of IA in nonneutropenic patients, Zhou et al. ${ }^{26}$ found a sensitivity of serum GM of $37.8 \%$ and a specificity of $87.1 \%$, with a positive predictive value of only $60.8 \%$. (Table 1 )

False positivity of GM assay may ocur by numerous factors. GM is found in many fungi and bacteria and can cause false positive results. Cross-reactivity with some beta-lactam antibiotics (piperacillin-tazobactam, amoxicillin-clavulanate) and with GM from other fungal species (Fusarium, Penicillium, Cladosporium, Histoplasma, Blastomyces, Paracoccidioides, Cryptococcus, Nigrospora, Paecilomyces, Trichothecium, Lichtheimia ramosa, and Geotrichum), as well as the epidemiological and biological factors are the conditions associated with $\mathrm{it}^{27}$. The proposed mechanism is that galactomannan in foods or bacteria having cross-reactive epitopes may translocate across the intestinal mucosa during periods of impaired mucosal integrity ${ }^{11}$. The sensitivity of detecting galactomannan in serum is decreased with the concurrent administration of mold-active antifungal therapy, for instance in patients receiving posaconazole or voriconazole prophylaxis which may prevent the circulation of $\mathrm{GM}^{28,29}$. In the study of Vena et al. ${ }^{30}$ investigating the performance of the routine serum GM in the diagnosis of IA in high-risk haematology patients receiving prophylaxis with micafungin; Surveillance of asymptomatic patients receiving prophylaxis with micafungin using GM was found to be unnecessary, because the results were either negative or false-positive. GM testing was recommended in the diagnosis of breakthrough IA in symptomatic patients during prophylaxis.

False-positive results are more likely to occur during the first 100 days following HCT and in patients with gastrointestinal tract mucositis caused by chemotherapy or graft-versus-host disease (GVHD) ${ }^{31}$. Levels of serum GM were detected to increase up to 4.5 for a few days immediately after the infusion of stem cells in a recent study ${ }^{24}$. In neonates and infants, immaturity of the intestinal mucosa may lead to the translocation of lipoglycans of Bifidobacterium, resulting in false-positive GM antigenemia ${ }^{27}$. While high false positive rates were reported in pediatric patients, several recent studies have shown its usefulness in children and similar results to adult patients ${ }^{5,32,33,34}$.

\section{GM Testing in Bronchoalveolar Lavage Fluid}

Detecting GM in BAL is a proposed tool to diagnose invasive pulmonary aspergillosis but the diagnostic value of BAL GM in children is rarely reported. A recommended cutoff by the manufacturer, a cutoff of 1 is used for BAL specimens ${ }^{35}$. It was shown that the GM levels appeared earlier in BAL fluid as compared to serum.

The FDA considers an OD index of $\geq 0.5$ to be positive for GM EIA in both serum and BAL fluid. In a prospective cohort study that included 530 patients with hematologic malignancy who had signs or symptoms of IFI, 155 (29\%) had a positive BAL GM result using an OD index threshold $\geq 0.5^{36}$. BAL GM had a sensitivity of $50 \%$ and a specificity of $73 \%$ for detecting proven or probable IA.

Sensitivities of BAL GM have been reported to be ranging 60 to $100 \%$ and specificities from $87.8 \%$ to $100 \%$ in several adult studies using a cutt-off index of $\geq 1.0^{37,38,39}$. Bergeron et al. ${ }^{40}$ reported a sensitivity and a specificity of $57.6 \%$ and $95.6 \%$ respectively when using a cut-off index of $\mathrm{BAL} \mathrm{GM} \geq 0.5$. A study including also immunocompetant patients reported sensitivity of $78 \%$ and specifity of $92 \%$ at a cut-off index of 0.98 in BAL in children ${ }^{41}$. (Table 1)

Sensitivity, specificity, positive predictive value, and negative predictive value of BAL GM for a diagnosis of proven and probable IPA were found to be $4 \%, 87.5 \%$, $82.4 \%$, and $87.5 \%$ in children in the study of de Mol M and et al. ${ }^{42}$. A significant relation was found for BAL GM and abnormal chest tomography. BAL GM and serum GM correlated significantly.

In non-neutropenic patients, IA is not always associated with angioinvasion. Symptoms of the disease are often nonspecific, typical radiological findings may be absent. Different from serum GM, BAL GM detection have been proven to be more advantageous in the non-neutropenic population and provides a great utility for diagnosis ${ }^{43,44,45}$.

BAL GM sampling as a prognostic tool is not established and in vivo research suggests that it is not possible ${ }^{46-50}$. Although serum GM sampling during therapy to monitor response is highly useful, utility for BAL GM for the same purpose is not suggested.

Similar to serum samples, false positivity can constitue 
a problem in BAL samples. False-positive results can occur, especially when the fluid that is used for BAL washes is contaminated with galactomannan and also common due to colonization as occurs in lung transplant recipients.

\section{GM Testing in Other Specimens}

GM can also be detected in cerebrospinal fluid (CSF), pleural fluid, sputum or urine, the experience with GM detection in these specimens are reported to be insufficient so there are no enough specific recommendations ${ }^{35}$.

\section{Conclusion}

The incidence rates of IFI and IA are increasing day by day. Diagnosis of IA is challenging; clinical symptoms are often nonspecific and classical diagnosis is difficult and rare. Diagnostic tools in the mycology for IFI ara limited currently. The GM test is available in many centers around the world and it should be used to help diagnosis. However the low positive predictive value should be considered.

\section{References}

1. Rubio PM, Sevilla J, Gonzalez-Vicent M, et al. Increasing incidence of invasive aspergillosis in pediatric hematology oncology patients over the last decade: a retrospective single centre study. J Pediatr Hematol Oncol. 2009; 31: 642-6.

2. Kontoyiannis DP, Marr KA, Park BJ, et al. Prospective surveillance for invasive fungal infections in hematopoietic stem cell transplant recipients, 2001-2006: overview of the Transplant-Associated Infection Surveillance Network (TRANSNET) Database. Clin Infect Dis. 2010; 50: 1091-100.

3. Babor F, Schuster F, Mackenzie C, et al. Invasive aspergillosis in pediatric oncology patients: a rare event with poor prognosiscase analysis to plan better targeted prophylactic or therapeutic measurement. Klin Padiatr. 2012; 224: 160-5.

4. Herbrecht R, Denning DW, Patterson TF, et al. Voriconazole versus amphotericin B for primary therapy of invasive aspergillosis. N Engl J Med. 2002; 347: 408-15.

5. Steinbach WJ, Marr KA, Anaissie EJ, et al. Clinical epidemiology of 960 patients with invasive aspergillosis from the PATH Alliance registry. Infect. 2012; 65: 453-64.

6. Sun KS, Tsai CF, Chen SC, et al. Galactomannan Testing and the Incidence of Invasive Pulmonary Aspergillosis: A 10-Year Nationwide Population-Based Study in Taiwan. PLoS One. 2016; 11(2).

7. Miceli MH, Grazziutti ML, Woods G, et al. Strong correlation between serum Aspergillus galactomannan index and outcome of aspergillosis in patients with hematological cancer: clinical and research implications. Clin Infect Dis. 2008; 46: 1412-22.

8. De Pauw B, Walsh TJ, Donnelly JP, et al. Revised definitions of invasive fungal disease from the European Organization for Research and Treatment of Cancer/Invasive Fungal Infections Cooperative Group and the National Institute of Allergy and Infectious Diseases Mycoses Study Group (EORTC/MSG) Consensus Group. Clin Infect Dis. 2008; 46: 1813-21.

9. Lehrnbecher T, Phillips R, Alexander S, et al. Guideline for the management of fever and neutropenia in children with cancer and/ or undergoing hematopoietic stem-cell transplantation. J Clin Oncol. 2012; 30: 4427-4438.

10. Groll AH, Castagnola E, Cesaro S, et al. Fourth European Conference on Infections in Leukaemia (ECIL-4): Guidelines for diagnosis, prevention, and treatment of invasive fungal diseases in paediatric patients with cancer or allogeneic haemopoietic stem-cell transplantation. Lancet Oncol. 2014; 15: 327-340.

11. Mennink-Kersten MA, Donnelly JP, Verweij PE. Detection of circulating galactomannan for the diagnosis and management of invasive aspergillosis. Lancet Infect Dis. 2004; 4: 349-357.

12. Cordonnier C, Pautas C, Maury S, et al. Empirical versus preemptive antifungal therapyfor high-risk, febrile, neutropenic patients: a randomized, controlled trial. Clin Infect Dis. 2009; 48: 1042-1051.

13. Maertens J, Theunissen K, Verhoef G, et al. Galactomannan and computed tomography-based preemptive antifungal therapy in neutropenic patients at high risk for invasive fungal infection: a prospective feasibility study. Clin Infect Dis. 2005; 41: 1242-1250.

14. Koo S, Bryar JM, Baden LR, et al. Prognostic features of galactomannan antigenemia in galactomannanpositive invasive aspergillosis. J Clin Microbiol. 2010; 48: 1255-1260.

15. Park SH, Choi SM, Lee DG, et al. Serum galactomannan strongly correlates with outcome of invasive aspergillosis in acute leukaemia patients. Mycoses. 2011; 54: 523-30.

16. Woods G, Miceli MH, Grazziutti ML, et al. Serum Aspergillus galactomannan antigen values strongly correlate with outcome of invasive aspergillosis: a study of 56 patients with hematologic cancer. Cancer. 2007; 110: 830-4.

17. Pfeiffer CD, Fine JP, Safdar N. Diagnosis of invasive aspergillosis using galactomannan assay: a meta-analysis. Clin Infect Dis. 2006; 42: 1417-27.

18. Limper AH, Knox KS, Sarosi GA, et al. An official American Thoracic Society statement: Treatment of fungal infections in adult pulmonary and critical care patients. Am J Respir Crit Care Med. 2011; 183: 96128.

19. Koo S, Bryar JM, Baden LR, et al. Prognostic features of galactomannan antigenemia in galactomannan-positive invasive aspergillosis. J Clin Microbiol. 2010; 48: 1255.

20. Han SB, Kim SK, Lee JW, et al. Serum galactomannan index for early prediction of mortality in immunocompromised children with invasive pulmonary aspergillosis. BMC Infect Dis. 2015 Jul 14; 15: 271.

21. Fisher BT, Zaoutis TE, Park JR, et al. Galactomannan antigen testing for diagnosis of invasive aspergillosis in pediatric hematology patients. J Pediatric Infect Dis Soc. 2012; 1: 103-111.

22. Walsh TJ, Anaissie EJ, Denning DW, et al. Infectious Diseases Society of America. Treatment of aspergillosis: clinical practice guidelines of the Infectious Diseases Society of America. Clin Infect Dis. 2008; 46: 327-360.

23. Freifeld AG, Bow EJ, Sepkowitz KA, et al. Clinical practice guidelinefor the use of antimicrobial agents in neutropenic patients with cancer: 2010 Update by the Infectious Diseases Society of America. Clin Infect Dis. 2011; 52: 427-431.

24. Gefen A, Zaidman I, Shachor-Meyouhas Y, et al. Serum galactomannan screening for diagnosis of invasive pulmonary aspergillosis in children after stem cell transplantation or with high-risk leukemia. Pediatr Hematol Oncol. 2015; 32(2): 146-52.

25. Cordonnier C, Botterel F, Ben Amor R, et al. Correlation between galactomannan antigen levels in serum and neutrophil counts in haematological patients with invasive aspergillosis. Clin Microbiol Infect.2009; 15(1): 81-6.

26. Zhou W, Li H, Zhang Y, et al. Diagnostic value of galactomannan antigen test in serum and bronchoalveolar lavage fluid samples from patients with nonneutropenic invasive pulmonary aspergillosis. J Clin Microbiol. 2017; 55: 2153-2161.

27. Mennink-Kersten MA, Ruegebrink D, Klont RR, et al. Bifidobacterial lipoglycan as a new cause for false-positive Platelia Aspergillus 
enzyme-linked immunosorbent assay reactivity. J Clin Microbiol. 2005; 43: 3925-31.

28. Duarte RF, Sánchez-Ortega I, Cuesta I, et al. Serum galactomannanbased early detection of invasive aspergillosis in hematology patients receiving effective antimold prophylaxis. Clin Infect Dis. 2014; 59 1696.

29. Cornely OA. Galactomannan testing during mold-active prophylaxis. Clin Infect Dis. 2014; 59: 1703.

30. Vena A, Bouza E, Álvarez-Uría A, et al. The misleading effect of serum galactomannan testing in high-risk haematology patients receiving prophylaxis with micafungin. Clin Microbiol Infect. 2017; 23(12): 1000.

31. Asano-Mori Y, Kanda Y, Oshima K, et al. False-positive Aspergillus galactomannan antigenaemia after haematopoietic stem cell transplantation. J Antimicrob Chemother. 2008; 61: 411.

32. Hayden R, Pounds S, Knapp K, et al. Galactomannan antigenemia in pediatric oncology patients with invasive aspergillosis. Pediatr Infect Dis J. 2008; 27: 815-9.

33. Castagnola E, Furfaro E, Caviglia I, et al. Performance of the galactomannan antigen detection test in the diagnosis of invasive aspergillosis in children with cancer or undergoing haemopoietic stem cell transplantation. Clin Microbiol Infect. 2010; 16: 1197-203.

34. Choi SH, Kang ES, Eo H, et al. Aspergillus galactomannan antigen assay and invasive aspergillosis in pediatric cancer patients and hematopoietic stem cell transplant recipients. Pediatr Blood Cancer 2013; 60: 316-22.

35. Marchetti O, Lamoth F, Mikulska M, et al. and the European Conference on Infections in Leukemia (ECIL) Laboratory Working Groups. ECIL recommendations for the use of biological markers for the diagnosis of invasive fungal diseases in leukemic patients and hematopoietic SCT recipients. Bone Marrow Transplantation. 2012; 47: 846-854.

36. Affolter K, Tamm M, Jahn K, et al. Galactomannan in bronchoalveolar lavage for diagnosing invasive fungal disease. Am J Respir Crit Care Med. 2014; 190: 309.

37. Clancy CJ, Jaber RA, Leather HL, et al. Bronchoalveolar lavage galactomannan in diagnosis of invasivepulmonary aspergillosis among solid-organ transplant recipients.J Clin Microbiol. 2007; 45: 1759-1765.14.

38. Husain S, Paterson DL, Studer SM, et al. Aspergillus galactomannan antigen inthe bronchoalveolar lavage fluid for the diagnosis of invasiveaspergillosis in lung transplant recipients. Transplantation. 2007; 83: 1330-1336.15.

39. Maertens J, Maertens V, Theunissen K, et al. Bronchoalveolar lavage fluid galactomannan forthe diagnosis of invasive pulmonary aspergillosis in patientswith hematologic diseases. Clin Infect Dis. 2009; 49: 1688-1693.

40. Bergeron A, Belle A, Sulahian A, et al. Contributionof galactomannan antigen detection in BAL to the diagnosis ofinvasive pulmonary aspergillosis in patients with hematologicmalignancies. Chest. 2010; 137: 410-415.

41. Desai R, Ross LA, Hoffman JA. The role of bronchoalveolarlavage galactomannan in the diagnosis of pediatric invasiveaspergillosis. Pediatr Infect Dis J. 2009; 28: 283-286.

42. de Mol M, de Jongste JC, van Westreenen M, et al. Diagnosis of invasive pulmonary aspergillosis in children with bronchoalveolar lavage galactomannan. Pediatr Pulmonol. 2013; 48(8): 789-96.

43. Cordonnier C, Botterel F, Ben Amor R, et al. Correlation between galactomannan antigen levels in serum and neutrophil counts in haematological patients with invasive aspergillosis. Clin Microbiol Infect. 2009; 15(1): 81-6.

44. Pasqualotto AC, Xavier MO, Sanchez LB, et al. Diagnosis of invasive aspergillosis in lung transplant recipients by detection of galactomannan in the bronchoalveolar lavage fluid. Transplantation. 2010; 90(3): 306-311.

45. Zou M, Tang L, Zhao S, et al. Systematic review and meta-analysis of detecting galactomannan in bronchoalveolar lavage fluid for diagnosing invasive aspergillosis. PLoS ONE. 2012; 7(8): e43347.

46. Kovanda LL, Desai AV, Hope WW. Prognostic value of galactomannan: current evidence for monitoring response to antifungal therapy in patients with invasive aspergillosis. J Pharmacokinet Pharmacodyn. 2017; 44: 143-151.

47. Hope WW, Kruhlak MJ, Lyman CA, et al. Pathogenesis of aspergillus fumigatus and the kinetics of galactomannan in an in vitro model of early invasive pulmonary aspergillosis:implications for antifungal therapy. J Infect Dis. 2007; 195: 455-466. .

48. Kovanda LL, Kolamunnage-Dona R, Neely M, et al. Pharmacodynamics of isavuconazole for invasive mold diesase: role of galactomannan for real-time monitoring of therapeutic response. Clin Infect Dis. 2017; 64: 1557-1563.

49. Mercier T, Guldentops E, Lagrou K, et al. Galactomanan, a surrogate marker for outcome in invasive aspergillosis: finally coming of age. Front Microbiol. 2018. doi:10.3389/fmicb.2018.00661.

50. Avcu G, Karapinar DY, Akinci AB, et al. Utility of the serum galactomannan assay fort he diagnosis of invasive aspergillosis in children with acute lymphoblastic leukemia. J Infect Dis. 2017; 54: 8-12 